\title{
Primary malignant neoplasms associated with chronic lymphocytic leukaemia
}

\author{
M. Lishner, M. Prokocimer, E. Ron ${ }^{1}$ and M. Shaklai \\ Division of Hematology, Beilinson Medical Center, Petah Tiqva and ${ }^{1}$ Department of Clinical Epidemiology, Sheba \\ Medical Center, and the Sackler Faculty of Medicine, Tel Aviv University, Tel Aviv, Israel.
}

\begin{abstract}
Summary: The relationship between chronic lymphocytic leukaemia (CLL) and primary malignant neoplasms was evaluated using data from the Hematology Division in Beilinson Medical Center and the Israel Cancer Registry.

The study population consisted of 81 patients diagnosed between 1962 and 1984. A total of 16 patients were found to have 21 malignant neoplasms in addition to their CLL. Excluding patients with nonmelanoma skin tumours, a 1.7 increased risk (statistically not significant) for developing second malignant neoplasms in CLL patients was detected.

The only tumour which occurred significantly more than expected subsequent to CLL diagnosis was brain cancer. The coexistence of multiple cancers in the same patient was diagnosed in four of the patients.

The results of this study further support the hypothesis that patients with CLL are prone to develop second neoplasms.
\end{abstract}

\section{Introduction}

The relationship between chronic lymphocytic leukaemia (CLL) and primary malignant neoplasms has been evaluated by several studies. An increased incidence of malignant neoplasms has been reported by some investigators ${ }^{1,2}$ but disputed by others. ${ }^{3,4}$ To explore further this association, we evaluated retrospectively 81 patients with CLL treated by the Hematology Division of Beilinson Medical Center. The results of this study are reported below.

\section{Materials and methods}

A series of 81 patients with CLL, diagnosed between 1962 and 1984, were identified from the records of the Hematology Division of Beilinson Medical Center. The criterion for diagnosis of CLL was a sustained and absolute lymphocytosis of $10 \times 10^{9}$ cells/ 1 in the peripheral blood. ${ }^{5}$ Bone marrow aspiration and/or biopsy was not done in every patient and was not a prerequisite for the diagnosis. The majority of patients received therapy which included alkylating agents (chlorambucil or cyclophosphamide) and prednisone. All 63 live patients are still in active follow-up at the Hematology Department.

The observed number of cancers among the study

Correspondence: M. Prokocimer M.D.

Accepted: 8 October 1986 population was compared to the expected number of cancers, based on data from the Israel Cancer Registry. Expected values were derived from age, sex, ethnic origin, calender-year and site-specific national cancer rates. ${ }^{6}$ Non-melanoma skin cancers are not reported to the Cancer Registry and were therefore excluded from the analysis. The ratio of observed to expected cases and $95 \%$ Miettemen exact confidence limits were calculated, assuming that the observed cases were distributed as a poisson variate. ${ }^{7}$

To study cancers appearing subsequent to CLL diagnosis, person-years at risk were calculated from date of CLL diagnosis to date of diagnosis of a second primary, date of death or 31 December 1984, whichever occurred first. Since CLL is a chronic disease and the exact time of diagnosis is dependent on many different factors, not all medically determined, we also evaluated multiple primaries occurring at any time during the patients' lives. For this analysis, personyears at risk were accumulated from 1960 (the year the Cancer Registry was established) to date of death or 31 December, 1984, whichever occurred first.

\section{Results}

Eighty-one patients were studied (Table I). Sixty-three $(77.8 \%)$ of the patients were born in Europe or America, $16(19.8 \%)$ in Asia or Africa and $2(2.5 \%)$ in Israel.

A total of 16 patients were found to have 21 
Table I Descriptive characteristics of study population

\begin{tabular}{ll}
\hline Number of patients: & 81 \\
Male: & $37(45.7 \%)$ \\
Female: & $44(54.3 \%)$ \\
Mean age at CLL diagnosis & $62.5( \pm 9.2)$ \\
Person-years at risk: & $473(1883)^{*}$ \\
Mean number years of follow-up: & $5.8(23.2)^{*}$ \\
Mean age at second primary: & $63.4(61.4)^{*}$ \\
\hline
\end{tabular}

* First number refers to the analysis of malignancies occurring subsequent to CLL diagnosis; number in parentheses refers to the analysis of malignancies occurring any time after 1960 .

malignant neoplasms in addition to the CLL (Table II). There were three basal (BCC) and three squamous cell (SCC) skin cancers following CLL - a combined cumulative incidence of 1.27 per 100 person-years. Seven other malignancies were diagnosed after CLL, yielding a cumulative incidence of 1.48 per 100 personyears. The cumulative incidence of all cancers (including non-melanoma skin cancers) appearing subsequent to CLL was $2.7 / 10^{2}$. Eight additional cancers occurred before CLL diagnosis - a total incidence of 1.12 per 100 person-years or $0.8 / 10^{2}$ excluding the nonmelanoma skin cancers.

Among the 7 patients (excluding BCC and SCC) whose associated cancer was subsequent to CLL diagnosis two women had breast cancer and the other two females had brain tumours. The three men all had $\frac{2}{\Phi}$

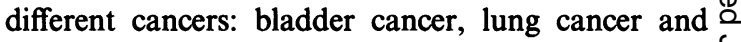
carcinoma with primary unknown. Comparing the $7 \stackrel{.}{\leftarrow}$ observed cancers to the 4.2 expected (Table III) $\stackrel{\vec{P}}{\vec{P}}$ yielded a ratio of 1.7 which was not statistically 7 significant. The ratio was similar for males (1.8) and $\frac{C}{0}$ females (1.6) and for European-American born (1.7) $\overline{\bar{c}}$ and Asian-African born (1.6). An increased risk of $\mathbb{\mathbb { D }}$ brain cancer $(O / E=20.0)$ was observed, but although $\frac{2}{2}$ the risk was statistically significant it was based on os only two cases.

Excluding patients having non-melanoma skin cancer, eleven had cancer which occurred either before or after CLL diagnosis, compared to 9.61 expected. Although the total number of observed cancers was very close to that expected $(\mathrm{O} / \mathrm{E}=1.1)$ significantly increased risks of melanoma $(\mathrm{O} / \mathrm{E}=14.3)$, carcinoma of the gastrointestinal tract $(\mathrm{O} / \mathrm{E}=4.2)$ and brain $(O / E=9.5)$ were seen.

\section{Discussion}

The coincidence of CLL and malignant tumours of the skin is well documented ${ }^{2,3,5}$ (Table IV). However, there $\overrightarrow{\vec{\theta}}$ is still controversy revolving around the question $\&$ whether patients with CLL also have an increased

Table II CLL patients with multiple cancers

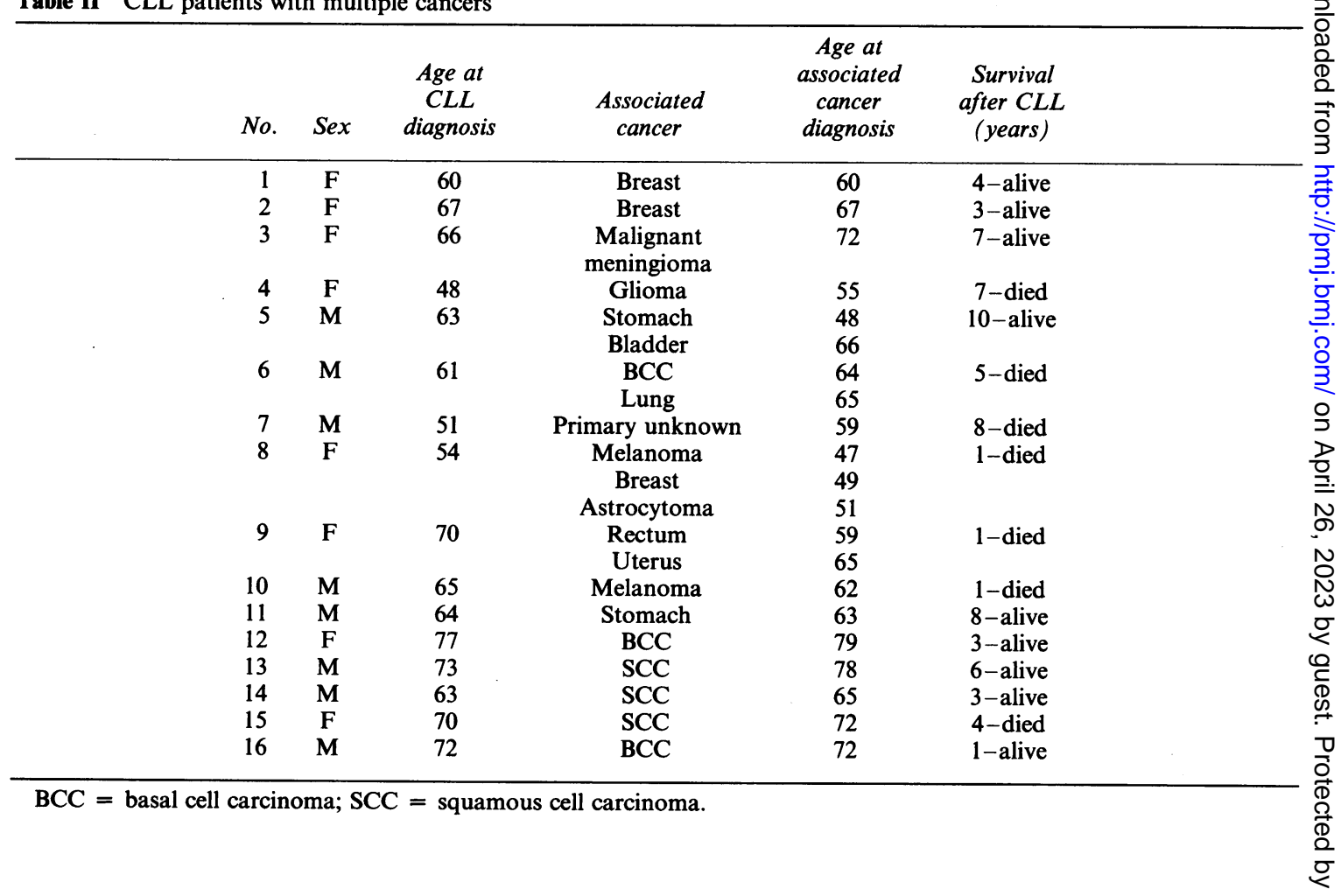


incidence of neoplasms, other than skin. Most studies have shown a higher than expected incidence, but many of the results were not statistically significant ${ }^{3,4,8,9}$ (Table IV). Although the numbers are small, the results from our study suggest that subsequent to CLL diagnosis, there is a slight, non-significant increase of second neoplasms over that expected. Since there is no registry of non-melanoma skin cancer in Israel, we were unable to conclude whether the 6 cases observed in our series is higher than expectation. The incidence of 1.27 per 100 person-years of risk is within the range of 0.73 to 3.38 observed in other studies (Table IV). The particularly high rate reported by Gunz \& Angus ${ }^{4}$ may be due to inflated rates of asymptomatic cancers diagnosed at the time of CLL diagnosis. It is also consistent with the relatively high incidence of melanoma found in New Zealand compared to that reported in Israel, the United States and Canada. ${ }^{10}$

In our series, the only tumour which occurred significantly more than expected subsequent to CLL diagnosis was brain cancer. This association has not been reported previously in other studies. Significantly increased risks of melanoma and gastrointestinal tract tumours were also noted by us. However, they occurred before CLL diagnosis. The increase in second malignancies in other series was attributed to an excess of cases of malignant melanoma, soft tissue sarcoma, and lung carcinomas. ${ }^{1}$

It is interesting to note that the incidence of nonskin cancers was similar in many of the studies. The rate ranged from a low of 0.80 per 100 years of risk to 2.68 , and 5 of the 8 studies had incidence rates between 1.35 and 1.48 (Table IV).

The factors that may play an aetiological role in the development of a second primary malignancy in CLL patients are defective cellular and humoral immunity; ${ }^{1,11,12}$ and prolonged treatment with alkylating agents. ${ }^{9}$ To date, however, only acute leukaemia and bladder carcinoma definitely are associated with alkylating agent therapy. ${ }^{13,14}$ Immune suppression is clearly a factor in inducing neoplasms in animals. ${ }^{15}$ Four patients in our study group had multiple malignant tumours (patients nos. 5, 6, 8,9). The coexistence of multiple tumours in the same patient is rare and may be a chance finding, but, alternatively, it may relate to immunosuppression which exists in CLL patients, or a genetic predisposition to develop malignant tumours. ${ }^{16}$

The mean time interval from the diagnosis of CLL to the diagnosis of the second, non-haematopoietic neoplasm was reported in one study to be 59 months. ${ }^{9}$ Since the mean follow-up of our patients was 68 months, it is theoretically possible that a few more cases will be diagnosed in the near future.

In summary, excluding patients with non-melanoma skin tumours, a 1.7 increased risk (statistically not significant) for developing second malignant neoplasms in CLL patients was detected by us. The higher than expected occurrence of brain tumours in our patients has not been reported previously. Significantly increased risks of melanoma and carcinoma of gastrointestinal tract were seen. However, they occurred before CLL diagnosis.

The coexistence of multiple cancers in the same patient, although rare, was diagnosed in four of our patients. These results further support the hypothesis that patients with CLL are prone to develop second neoplasms.

Table III Observed and expected malignant neoplasms occurring among CLL patients

\begin{tabular}{lccc}
\hline Cancer site (ICD-9) & Observed (O) & Expected $(E)$ & $O / E(95 \% C I)$ \\
\hline I: Cancers occurring subsequent to CLL diagnosis & & $1.7(0.7-3.3)$ \\
All sites (140-209) & 7 & 4.17 & $2.6(0.1-12.6)$ \\
Lung (162) & 1 & 0.39 & $3.9(0.7-13.0)$ \\
Breast (174) & 2 & 0.51 & $4.5(0.2-22.4)$ \\
Bladder (188) & 1 & 0.22 & $20.0(3.4-66.1)$ \\
Brain (191-192) & 2 & 0.10 & $2.6(0.1-13.0)$ \\
Primary unknown & 1 & 0.38 & \\
II: Cancers occurring at any time* & & & $1.1(0.6-2.0)$ \\
All sites (140-209)* & 11 & & $4.2(1.1-11.3)$ \\
Gastrointestinal (151-154) & 3 & 9.61 & $1.1(0.5-5.2)$ \\
Lung (162) & 1 & 0.72 & $14.3(2.4-47.2)$ \\
Melanoma (172) & 2 & 0.95 & $1.5(0.3-4.9)$ \\
Breast (174) & 2 & 0.14 & $9.5(1.6-31.5)$ \\
Brain (191-192) & 2 & 1.34 & $1.3(0.7-6.5)$
\end{tabular}

\footnotetext{
* Patients with multiple primaries were categorized by site according to the first cancer, i.e., the patient with stomach and bladder cancer was included as a stomach cancer, the patient with melanoma, breast cancer and astrocytoma was classified as melanoma and the patient with rectum and uterus cancer as rectum.
}

ICD = International Classification of Diseases. 


\section{References}

1. Bartal, A., Bentwich, Z., Manny, N. \& Izac, G. Ethical and clinical aspects of chronic lymphocytic leukemia in ${ }^{\infty}$ Israel. A survey of 288 patients. Acta Haematol 1978, 60: $\overrightarrow{0}$ 161-171.

2. Manusow, D. \& Weiberman, B.H. Subsequent neoplasia $\vec{\omega}$ in chronic lymphatic leukemia. JAMA 1975, 232: 267269.

3. Berg, J.W. The incidence of multiple primary cancers. $J$. Natl Cancer Inst 1967, 38: 741-752.

4. Gunz, F.W. \& Angus, H.B. Leukemia and cancer in the same patient. Cancer 1965, 18: 145-152.

5. Chronic Leukemia-Myeloma Task Force Proposed guidelines for protocol studies. III. Chronic leukemias. ज़ Cancer Chemother Rep 1973, 4: 159-165.

6. Monson, R.R. Analysis of relative survival and proportional mortality. Comput Biomed Res 1974, 7: 325-332.

7. Rothman, K.J. \& Boice, J.D. Epidemiologic analysis with a programmable calculator. DHEW Publ No. (NIH) 79-1649. Washington, D.C., US Government $\overrightarrow{0}$ Printing Office, 1979.

8. Stavraky, K.M., Watson, T.A., White, D.F. \& Miles, E. Chronic lymphocytic leukemia and subsequent cancer the same patient. Cancer 1979, 26: 410-414.

9. Krause, J.R., Ayuyang, H.Q. \& Ellis, L.D. Secondary non-hematopoietic cancers arising following treatment of hematopoietic disorders. Cancer 1985, 55: 512-515. Ф

10. Waterhouse, J., Muir, C., Shanmugarasatnam, K. \& $\stackrel{2}{\vec{\prime}}$ Powell, J. (eds) Cancer Incidence in Five Continents. IARC Scientific Publication No.42. International Agency for Research on Cancer, Lyon, 1982, 4: 614615.

11. Greene, M.H., Hoover, R.N. \& Fraumeni, J.F. Sub- $\bar{\partial}$ sequent cancer in patients with chronic lymphocytic 3 . leukemia. J Natl Cancer Inst 1978, 61: 337-340.

12. Moayeri, H., Han, T., Stutzman, L. \& Sokal, J.E. Second neoplasms with chronic lymphocytic leukemia. NY State $ᄋ$ J Med 1976, 76: 378-381.

13. Casciato, D.A. \& Scott, J.L. Acute leukemia following prolonged cytotoxic agent therapy. Medicine 1979, 58: 32-47.

14. Valagussa, P., Santoro, A., Kenda, R., Fossati-Bellani, E., Franchi, F., Banfi, A., Rilke, F. \& Bonadonna, G. N Second malignancies in Hodgkin's disease: a complication linked to certain forms of treatment. $\mathrm{Br} M e d \mathrm{~J} 1980$, 280: $216-219$.

15. Cole, L.Z. \& Novel, P.C. Immunological factors modifying the induction of neoplasms in irradiated mice. Proceedings of the IV Perugia Quadrennial International $\stackrel{0}{\leftarrow}$ Conference on Cancer, Perugia University. Ed. Locio \& Severins, Perugia, Italy, 1970.

16. Kornberg, A., Matzner, Y. \& Polliack, A. Hodgkin's $\frac{T}{T}$ disease and subsequent chronic lymphocytic leukemia in $\frac{\mathrm{O}}{\mathbb{D}}$ a patient with breast carcinoma. Israel J Med Sci 1978, 14: $1242-1246$. 